\title{
Fog Computing Infrastructure Simulation Toolset Review for Energy Estimation, Planning and Scalability
}

\author{
Kelvin N. Lawal, Titus K. Olaniyi, Ryan M. Gibson \\ School of Computing, Engineering and Built Environment \\ Glasgow Caledonian University, United Kingdom
}

\begin{abstract}
The exponential growth of smart Internet of Things (IoT) devices has led to an increase in data generation, transfer, storage, and management within IoT infrastructures. Consequently, there is an increase in latency, network bandwidth usage and energy consumption for managing data. This paper evaluates energy efficiency improvements through extending the traditional Cloud computing continuum with the introduction of the Fog computing paradigm. Using proof of concept (PoC), this research addresses some of the challenges with traditional cloud computing such as high energy consumption with network simulation toolsets. An evaluation was conducted to determine the best suited network simulation software tool for critically analysing Fog computing and traditional Cloud computing in terms of data management, planning and energy efficiency. This paper demonstrates a reduction in energy consumption with the introduction of Fog computing layer technologies when compared to traditional Cloud computing architecture through the iFogSim network simulation toolset. Furthermore, the differences in energy consumption and data management with Fog and Cloud computing data centres are explored.
\end{abstract}

Keywords: Energy Efficiency; Fog Computing; Cloud Computing; Data Management; Network Simulation Software Toolsets.

\section{Introduction}

The network simulation software evaluation process identifies fourteen network simulator software toolsets for Cloud, Edge and Fog environments. The evaluation exercise used a set of pre-screened and carefully selected attributes to compare the selected tools to ensure the choice meets the requirements for conducting a simulation proof of concept (PoC) experiment to further achieve the research aims and objectives.

The process also reviewed key characteristics highlighting similarities and differences in order to justify the decision process of the selection exercise. Some of the key attributes used in the comparison process includes energy consumption module, sensor simulation, data management process capabilities, mobile nodes, network communication, scalability, graphical user interface (GUI), mobility, infrastructure and network level modelling, application-level modelling, and computing paradigm target system capabilities.

The exponential growth of smart Internet of Things (IoT) devices and use of Cloud services is trending in a period when mounting emphasis is being put on the need to improve energy consumption to reach net zero energy [1]. According to [2], there is a rapidly growing demand for data centre services due to the increase in global data centre traffic including computing instances and workloads, which is expected to further increase in the coming years. In major economies there is a drive to ensure measures and policies are in place to support energy efficiency in multiple economic sectors. where ICT and data centres are of particular importance [3]. Consequently, the integration of energy efficiency adoption into data centres is crucial to the future development of energy systems and adherence. Fog and Edge computing research are still relatively in their infancy with regards to infrastructure data management and energy development therefore, the support of IoT, Fog and Edge computing through simulation frameworks is limited [4]. This paper explores current simulation toolsets for evaluating Fog and Cloud computing infrastructure data management with energy consumption.

This paper aims to conceptualise the benefits of Fog computing enabling the arrangement of services and applications within Fog nodes situated between the network Edge and the Cloud data centre. Extending infrastructure layers with the introduction of Fog and Edge resources allows services and applications to utilise various features such as efficient energy consumption, latency reduction and efficient network bandwidth usage.

\section{Literature review}

Cloud computing infrastructure can be defined as a model for facilitating on demand network access to a collective group of configurable computing resources that can be quickly provisioned and released 
with negligible service provider engagement or management effort [1]. The global deployment of a centralised Cloud computing architecture has become essential to store significant transmitted quantities of data within the IoT network. On the other hand, Cloud computing also has challenges such as high energy consumption which this paper aims to address using Edge and Fog computing paradigms.

Edge computing extends Cloud computing through a network layer with access to smart IoT devices, providing computing capabilities at the local level [5]. The introduction of Edge computing paradigm, a decentralised Cloud computing system which allows local assessment of Cloud services such as computing and storage capabilities at the Edge of the network closer to the data source [6]. As a result, Edge computing is revolutionising the way data is being generated, transferred, processed, stored and delivered from billions of smart devices through local level computing capabilities which improves energy efficiency within an IoT network.

We described in Figure 1, the introduction of a third tier Fog computing layer into the traditional two tier Cloud computing architecture between user device layer and the Cloud layer extends the services of Cloud computing to the Edge of the network [7]. Fog computing is an emergent architecture that enhances the distribution of services such as computation, storage, scheduling and networking capabilities closer to data sources along the Cloud-tothings continuum. Often the terms Fog and Edge are used interchangeably, although Fog is broader than the classic concept of Edge [8].

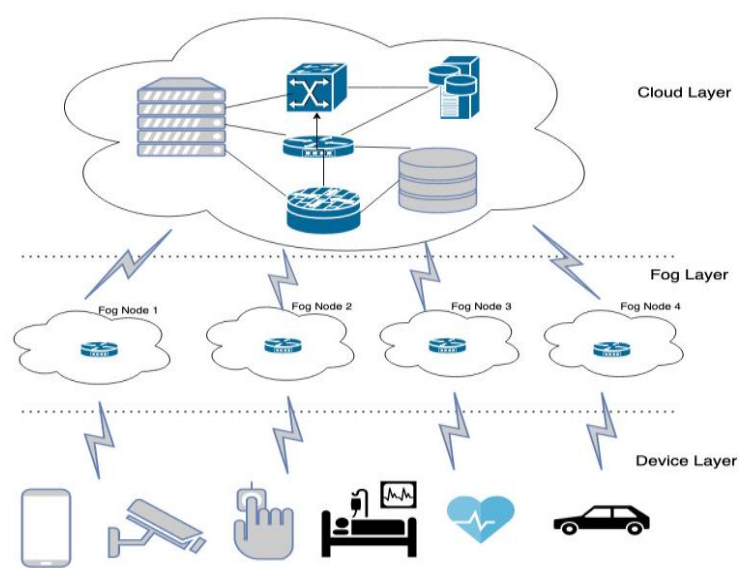

Figure 1. Three tier network architecture

Fog computing exploits the processing power of computing resources locally as opposed to the remote Cloud data centre processing, narrowing the geographical location between the terminal smart devices and processors thereby improving energy efficiency, transmission latency and network bandwidth usage [9].

\section{Data Management}

The concept of data management broadly refers to architectures, practices, and procedures for accurate management of data lifecycle needs with a particular system infrastructure [10]. Furthermore, [11] describes data management as an essential feature of IoT especially when billions of interconnected devices exchange data, the management of data generated from these interconnected devices becomes critical. The large increase in data generated from smart IoT devices are bandwidth intensive. As a result, the increase in data generation, transmission and storage from smart devices increase energy consumption levels within the IoT network.

\section{Energy Modelling}

According to [12], one of the most critical challenges in state-of-the-art data centres is the high energy consumption associated with Cloud computing elements. It is estimated that data centres currently consume over 70 billion $\mathrm{kWh}$ in the United States alone and is forecasted to increase to 73 billion $\mathrm{kWh}$ by the year 2020 [13]. In order to measure energy consumption in data centres, energy consumption models through Cloud and Fog infrastructure simulator tools are used. Although simulators have several advantages, there are also several limitations and challenges which are currently being addressed such as high energy consumption and scalability to manage increase in heterogeneous device. There are various approaches to modelling infrastructure network energy consumption, where a United States energy usage report on cloud data centre metrics [13] demonstrated a network energy consumption mathematical model derived from active port usage. Equation (1) measures the network energy consumption through variable active ports while considering port speed, power consumption and the number of hours on an annual basis.

$$
E_{y}=\sum_{s \in S} N_{s, y} * P_{s, y} * h_{y}
$$

Where:

$E_{y}=$ Represents measurement of network energy consumption annual

$\mathrm{S}=$ Represent variable port speed in (MB)

$N_{s, y}=$ Number of active ports speed annually

$P_{s, y}=$ Power consumption of ports and speed annually

$h_{y}=$ Number of hours annually 


\section{Methodology}

In this section, the introduction of Fog computing within the context of an IoT network is demonstrated through a PoC using the network simulation software toolset to demonstrate Fog computing advantages and how these advantages address Cloud computing limitations such as high energy consumption, latency and network bandwidth usage.

For the benefit of this paper, the network simulation software toolset evaluation exercise is conducted to select the best simulator to use as part of the method to model Fog and Cloud computing within an IoT network with a focus on energy consumption challenge and how Fog computing addresses this challenge.

\subsection{Network Simulation Software Toolset}

Various network simulation software toolsets with a number of features, functionalities, characteristics and input topology formats have been developed in line with the evolution of IoT, Cloud, Edge and Fog computing paradigms [14]. However, each toolset has advantages, disadvantages and trade-offs involved, which limits the capabilities of one toolset satisfying requirements for every type of network infrastructure scenario.

Testing in a real-world large scale network infrastructure is not practical, high-cost, with risk to impacting live services and applications. Therefore, using a network simulator software toolset to simulate a testbed or development environment is necessary and cost effective within large-scale network infrastructures to test novel solutions prior to launching in a production or live environment [15]. Consequently, determining the most suitable and appropriate network simulation tool can be quite a daunting task for researchers and could lead to severe delays, limitations or flawed simulations.

The simulator used to model and evaluate Cloud and Fog computing continuums should have certain characteristics and capabilities such as measuring latency, energy consumption and network bandwidth usage to fully represent realistic environments reflective of the outputs within a complex environment.

Completing the first stage of the evaluation exercise, three network simulation software toolsets were identified and chosen NetFogSim++, iFogSim and CupCarbon. Some of the attributes validated ranged from extent of programming to energy consumption measurement model inclusion as a function.

The Table 1 presents three network simulation software toolsets evaluated from literature which meet the requirement for energy consumption module attributes which is critical to evaluating infrastructure data management and energy efficiency.
Table 1. Network simulation software toolset evaluation table

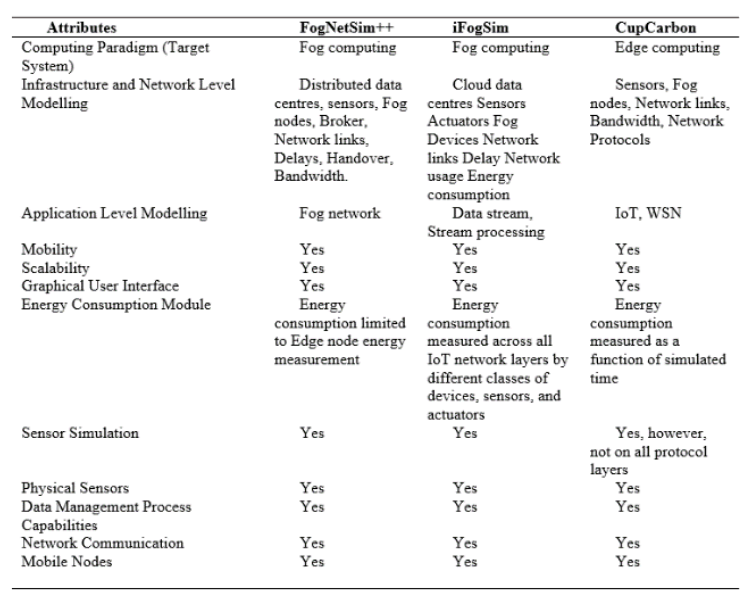

\section{Fog Computing Network Simulator Software Benefits and Challenges}

The following are some of the benefits of Fog computing network simulation software toolsets; the support of multiple features such as fault injection and the arbitrary removal of links and nodes, this allows for the variable mechanism tests for resilience, fault tolerance and complex experiments within realistic environments [15]. Most simulators also have monetary and energy consumption possibilities embedded within network simulator software toolsets. While other network simulators especially for Fog computing has dynamic capabilities such as variable service infrastructure, inclusion and exclusion of more resources on demand to support the dynamic nature of Fog computing environments [15]. There are more benefits to using a network simulator software toolset however, as a primary focus of this paper, most of the benefits mentioned are in line with and supports Fog computing environments.

The amplified mobile device ubiquity and the surge of network and computing end points over the decade through low cost sensors connections has certainly been on the increase. The traditional Cloud computing network infrastructure was not designed with the capacity to manage compute, processing and storage for billions of devices with multiple end points and irregular network connection in a dynamic environment [16]. In addition, the profound complexity of design, test, implementation and heterogeneous formats of data sources from the sharp increase in number of sensors embedded within IoT devices will require a greater level of understanding and a steep learning curve to be able to model and simulate these environments. These challenges through lessons learnt serves to encourage the developmental review of a more improved design, reliability, fault tolerance and holistic network simulation software toolset modelling and framework. 
Additionally, current network simulation software toolsets have to manage a multitude of variable workloads due to increased number of devices consequently, there should be greater support for modelling heterogeneous data abstractions and network topological workflow, heterogeneous query and dataflows.

FogNetSim++ is a network simulation software toolset designed on the open source OMNeT++ framework and developed for Fog computing, enabling users to configure large scale Fog network simulations using detailed configuration options, extensive libraries and discrete events. Furthermore, FogNetSim++ allows users to simulate variable features of heterogeneous devices, such as handover to track data or device source using flexible design and the incorporation of bespoke algorithms through class extension [17]. Some key features of the network simulation software toolset evaluation specific include the energy consumption module which FogNetSim++ supports through extensive energy measure for each participating Fog nodes and mobile devices, and a range of mobility models.

iFogSim is a Java technology based state-of-theart simulation software toolset designed for Fog computing environment scenario experiments in assessing resource management, scheduling policies including impact on energy consumption, latency and network bandwidth usage measurement [18]. Furthermore, iFogSim has extensive capabilities to simulate and model various infrastructure scenarios and features such as RAM, transient storage capabilities, CPU and energy consumption modelling. As a Java based network simulation software toolset, iFogSim supports multiple java classes that corresponds to applications, sensors, actuators, tuples and Fog devices, Fog nodes and large-scale heterogeneous nodes. In addition, iFogSim has been extended to optimise data placements in IoT devices and Fog nodes to ensure the management of data placements in relation to various objectives such as improving energy consumption, latency and network bandwidth usage [19]. iFogSim, was designed with detailed energy consumption measurement capabilities across all layers of the network which is the focus of the PoC. Consequently, iFogSim is the best fit for measuring energy efficiency, latency and network bandwidth usage in an IoT network infrastructure.

CupCarbon is a Java based developed network simulation software toolset designed with easy to use configurable objects, multi agent capabilities with embedded wireless network simulator geolocation sensor which supports digital geographic interface such as OpenStreetMap and simulate sensor networks and modelling. CupCarbon main architecture comprises of about seven modules: 2D/3D city model module, Mobility module, Network module, Communication Script module, Radio Channel
Propagation module, Interference module and Simulation module [20]. The mobility, network, communication and simulation modules are relevant however, there are significant limitations such as the energy simulation module which is calculated as a function of simulated time and not an extensive analysis of the data management process. Furthermore, CupCarbon is developed and designed for Edge computing and does not support the Fog computing layer specific technology paradigm. As a result, CupCarbon is not a suitable network simulation software toolset for this paper.

Under NetFogSim++, the energy measurement is done through participating Fog nodes only excluding the sensor and actuator embedded device layer which limits the scale at which this simulator can model Fog capabilities while in iFogSim, energy consumption is measured across all the devices including Fog nodes, applications, tuples and sensors across all network computing layers. Where iFogSim provides a comprehensive and extensive energy consumption analysis through all computing layers involved within the infrastructure. However, with CupCarbon, energy consumption is measured as a function of the time it takes to transmit data to the Cloud computing layer. This lacks support for sensors, actuators, geographical mobility and other Fog modelling capabilities. Therefore, iFogSim network simulation software toolset is best suited to evaluate data management and energy consumption for Cloud and Fog computing infrastructures.

\section{Energy Consumption Estimation Analysis}

The simulation scenario modelled in Figures 2 and 3 is configured with data processed at 3 million instructions per second, uplink bandwidth of $1 \mathrm{~GB}$, downlink bandwidth of $1.5 \mathrm{~GB}$ while the sensors and actuators transmit data to the Fog node at the rate of 50 milli seconds.

iFogSim has a network GUI which visualises the topological models as shown in Figure 2 and 3 with the required parameters used to demonstrate the PoC. Furthermore, Figure 4 highlights energy consumption increase for Cloud data centre when data processing is routed to the Cloud while the opposite occurs when processing is routed through the Fog nodes (DC). However, the rate of change for DC Cloud and DC Fog also varies, with the level energy consumption increased for both DC Fog and DC Cloud as the configuration is scaled up however, at different rates as shown in Figure 4. The network topological configuration of 5 Fog nodes and 3 devices $(5,3)$ with an energy output measurement of $8072 \mathrm{KJ}$ and $16145 \mathrm{KJ}$ for DC Fog and DC Cloud respectively. This shows the rate of energy consumption increase for DC Cloud is roughly double the rate of increase for DC Fog. With regards to scalability, Figures 2 and 3 
describe increased scale of Fog nodes and devices from 1,3 to 5,5 and proportionate to an increase in energy consumption.

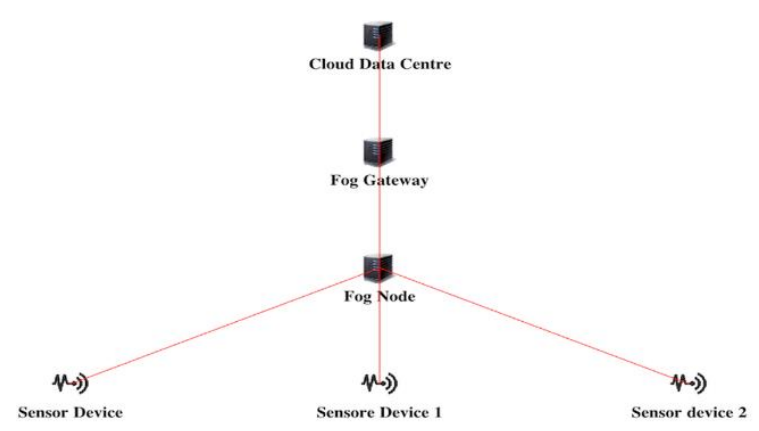

Figure 2. iFogSim network topology configuration 1 Fog node with 3 devices connected $(1,3)$

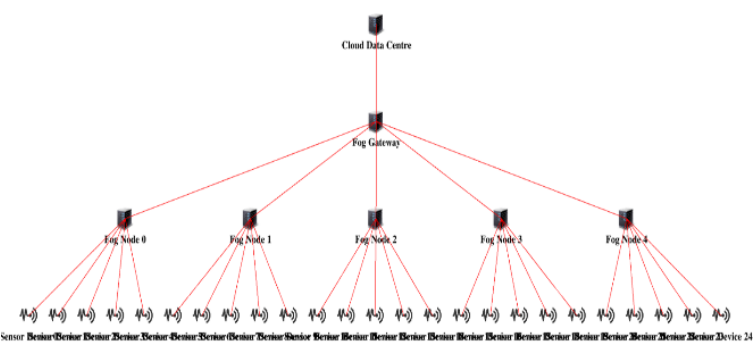

Figure 3. iFogim network topology configuration 5

Fog nodes with 5 devices connected $(5,5)$

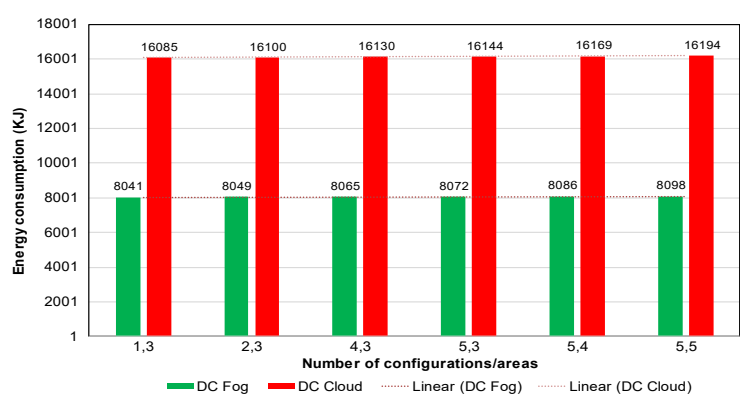

Figure 4. DC Fog and Cloud energy consumption combined output

\section{Results and Discussion}

The results demonstrate that Fog infrastructure is significantly more energy efficient than Cloud approaches when processing data and as network infrastructure scales. Based on linear trend analysis in Figures 5 and 6 , it can be observed that the energy consumption linear equations are $\mathrm{Y}=11.589 \mathrm{x}+8027.8$ and $Y=21.916 x+16061$ for DC Fog and DC Cloud.

The linear equations gradient parameter indicates the energy consumption rate of change as infrastructure increases. DC Fog and DC Cloud demonstrated energy consumption rate of change values of 11.589 and 21.916 respectively. Although

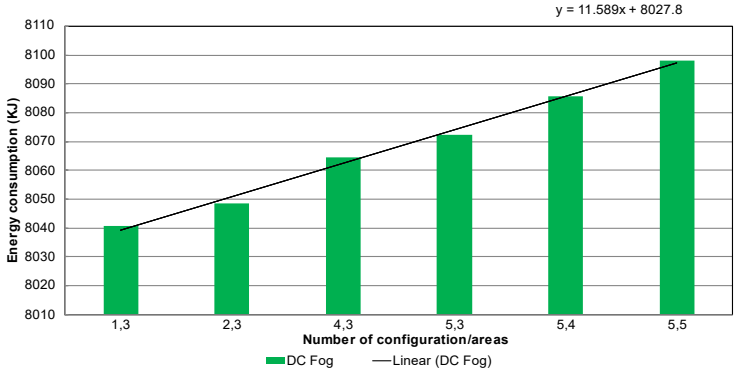

Figure 5. DC Fog energy consumption output

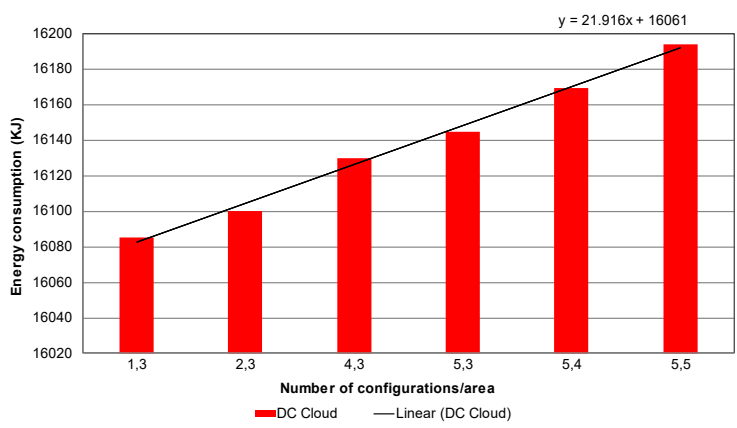

Figure 6. DC Cloud data centre energy consumption output

performance parameters demonstrate that both DC Fog and DC Cloud are linear. The rate of change for energy consumption in DC Fog data processing is 89\% lower compared to DC Cloud. Furthermore, as scale increases, DC Cloud increases at a higher rate of change of $62 \%$, making DC Fog a preferred option for energy savings. However, due to limited capabilities, only 5 user devices with up to $5 \times 10000=50000$ concurrent tasks can be computed at a single Fog node compared to DC Cloud based approach which is more scalable and can handle up to 30 end user devices with $30 \times 10000=300000$ tasks per node due to increased range of capacity.

\section{Conclusion}

The network simulation software toolset evaluation demonstrated iFogSim as the most suitable simulator toolset for investigating the energy consumption measurements for data management in an IoT network infrastructure. The PoC further demonstrates that the introduction of Fog computing layer to the IoT architecture extending the traditional Cloud computing infrastructure successfully reduces the energy consumption levels when data is processed through the Fog computing layer as opposed to the Cloud computing layer. There are other benefits of Fog computing alongside the various methods that can further reduce energy consumption and conform to some Sustainable Development Goals (SDG) such as improved scheduling in Fog nodes and the use of algorithms to enhance data management performance. 
The results of this paper can be expanded for further investigations into critical areas that can further improve energy efficiency with Fog computing and algorithms which can further improve latency and network bandwidth usage. Further studies should be conducted in these areas to reduce the impact of the plethora of smart IoT devices as they continue to grow at an exponential rate.

\section{References}

[1] Baliga, J., Ayre, R. W. A., Hinton, K. and Tucker. R. S. (2011). Green cloud computing: Balancing energy in processing, storage, and transport. Proceedings of the IEEE, vol. 99, no. 1, pp. 149-167 DOI: 10.1109/JPROC .2010 .2060451 .

[2] Andrae, A. (2018). Should we be concerned about the power consumption of ICT?,"Around the World Sustainable Research e-Conference, no. March, DOI: 10.13140/RG.2.2.10935.78247.

[3] Bertoldi, P. (2016). The European Code of Conduct for 2016.

[4] Khan, S., Parkinson, S. and Qin. Y. (2017). Fog computing security: a review of current applications and security solutions. Journal of Cloud Computing, vol. 6, no. 1, DOI: 10.1186/s13677-017-0090-3.

[5] Bendechache, M., Svorobej, S., Endo, P. T. and Lynn, T. (2020). Simulating resource management across the cloud-to-thing continuum: A survey and future directions. Future Internet, vol. 12, no. 6, pp. 1-25. DOI: 10.3390/FI12060095.

[6] Azar, J., Makhoul, A., Barhamgi, M., and Couturier, R. (2019). An energy efficient IoT data compression approach for edge machine learning. Future Generation Computer Systems, vol. 96, pp. 168-175, DOI: 10.1016/j.future .2019.02.005.

[7] Hu, P., Dhelim, S., Ning, H. and Qiu, T., (2017). Survey on fog computing: architecture, key technologies, applications and open issues. Journal of Network and Computer Applications, vol. 98, no. December 2018, pp. 27-42, DOI: 10.1016/j.jnca.2017.09.002.

[8] Chiang, M. and Zhang, T. (2016). Fog and IoT: An Overview of Research Opportunities. IEEE Internet of Things Journal, vol. 3, no. 6, pp. 854-864, DOI: 10.1109/JIOT.2016.2584538.

[9] Liu, Y., Fieldsend, J. E. and Min, G. (2017). A framework of fog computing: Architecture, challenges, and optimization. IEEE Access. vol. 5, pp. 25445-25454, DOI: 10.1109/ACCESS.2017.2766923.

[10] M. Abu-Elkheir, M. Hayajneh, and N. A. Ali, "Data management for the Internet of Things: Design primitives and solution," Sensors (Switzerland), vol. 13, no. 11, pp. 15582-15612, 2013, doi: 10.3390/s131115582.
[11] O. Vermesan and P. Friess, Internet of Things: Converging Technologies for Smart Environments and Integrated Ecosystems. 2013.

[12] Ahvar, E., Orgerie, A. C. and Lebre, A. (2020). Estimating Energy Consumption of Cloud, Fog and Edge Computing Infrastructures," IEEE Transactions on Sustainable Computing, 2020, DOI: 10.1109/TSUSC.2019 .2905900 .

[13] Shehabi A. et al., (2016). OSTI United States Data Centre Energy Usage 2016. https://www.osti.gov/ servlets /purl/1372902 (Access Date: 7 June 2021).

[14] Fakhfakh, F., Kacem, H. H., and Kacem, A. H., "Simulation tools for cloud computing: A survey and comparative study," Proceedings - 16th IEEE/ACIS International Conference on Computer and Information Science, ICIS 2017, pp. 221-226, 2017, DOI: 10.1109/ICIS.2017.7959997.

[15] Perez Abreu, D., Velasquez, K., Curado, M., and Monteiro, E. (2019). A comparative analysis of simulators for the Cloud to Fog continuum. Simulation Modelling Practice and Theory, vol. 101, no. October, p. 102029, DOI: 10.1016/j.simpat.2019.102029.

[16] Svorobej S. et al. (2019). Simulating fog and edge computing scenarios: An overview and research challenges. Future Internet, vol. 11, no. 3, pp. 1-15, DOI: 10.3390/fi11030055.

[17] Qayyum, T., Malik, A. W., Khattak, M. A. K., Khalid, O. and Khan, S. U. (2018). FogNetSim++: A Toolkit for Modeling and Simulation of Distributed Fog Environment. IEEE Access. vol. 6, no. May 2019, pp. 63570-63583, 2018, DOI: 10.1109/ACCESS.2018.2877696.

[18] Gupta, H., Vahid Dastjerdi, A., Ghosh, S. K. and Buyya, R. (2017). iFogSim: A toolkit for modeling and simulation of resource management techniques in the Internet of Things, Edge and Fog computing environments. Software - Practice and Experience. vol. 47, no. 9, pp. 12751296, 2017, DOI: 10.1002/spe.2509.

[19] Naas, M., Boukhobza, I., Raipin, J., Parvedy, P. and Lemarchand, L. (2018). An Extension to iFogSim to Enable the Design of Data Placement Strategies. 2018 IEEE 2nd International Conference on Fog and Edge Computing, ICFEC 2018 - In conjunction with 18th IEEE/ACM International Symposium on Cluster, Cloud and Grid Computing, IEEE/ACM CCGrid 2018, pp. 1-8, 2018, doi: 10.1109/CFEC.2018.8358724.

[20] Mehdi, K., Lounis, M., Bounceur, A. and Kechadi, T. (2014). CupCarbon: A multi-agent and discrete event Wireless Sensor Network design and simulation tool. SIMUTools 2014 - 7th International Conference on Simulation Tools and Techniques. pp. 126-131, 2014, DOI:10.4108/icst.simutools.2014. 254811. 